\section{GW23-e2107 FUNCTIONAL EXPRESSION OF TSH RECEPTOR IN THE VENTRICLE OF RATS AND MICE: INVOLVEMENT A REGULATION OF HMGCR BY TSH VIA THE CAMP/PKA/ PCREB SIGNALLING PATHWAY}

doi:10.1136/heartjnl-2012-302920a.25

${ }^{1}$ Huang Wen, ${ }^{2}$ Zhao Jiajun. ${ }^{1}$ Qian Foshan Hospital of Shandong University; ${ }^{2}$ Provincial Hospital of Shandong University

Objectives Elevated thyroid-stimulating hormone (TSH) and hypercholesterolemia commonly coexist, as typically seen in subclinical hypothyroid, which can lead to and aggravate heart disease if it gets serious. However, there is no known whether TSH plays direct action on the heart. In the present study, we investigated the expression and function of thyrotropin receptor (TSHR) in ventricle tissue and myocytes. We further examined that TSH regulated the expression of 3-hydroxy-3-methyl-glutaryl coenzyme A reductase (HMGCR), a rate-limiting enzyme in cholesterol synthesis, by interacting with TSHR in ventricular myocytes. Our research provides a new insight into explore the mechanism of hypothyroid cardiomyopathy.

\section{Methods}

(1) Rat ventricle myocyte cell line $\mathrm{H} 9 \mathrm{C} 2$ cells, ventricle tissues of normal Wistar rats as well as BALB/c and C57BL/6J mice were selected as research samples. Rat thyroid cell line FRTL-5 cells were used as the positive controls. TSHR mRNA expression was measured using Reverse-Transcription (RT); While the expression of TSHR protein was measured using western blotting and immunofluorescence, respectively.

(2) After starved in 2\% CS-FBS medium to achieve synchronisation, H9C2 cells were stimulated with bTSH for the different time and concentrations, collected the cells and tested the intracellular cAMP production, HMGCR mRNA and protein expression, respectively. At the same time, as the secreted contents of brain natriuretic peptide (BNP) in the culture media were assayed and the corresponding cell protein level was also collected to correct the BNP levels.

(3) H9C2 cells were pre-incubated with the adenylyl cyclase inhibitor SQ22536 or cAMP-dependent protein kinase A (PKA) inhibitor $\mathrm{H} 89$ respectively before subsequent stimulation with $\mathrm{bTSH}$ for $48 \mathrm{~h}$. The intracellular cAMP production and the expression of pCREB and HMGCR were examined, respectively.

(4) To further investigate the role of TSH in the regulation of HMGCR in the ventricle in vivo, we employed $\mathrm{Tshr}^{-/-}$type 
(supplement with exogenous T4) and wild type C57BL/6 mice. Testing the expression of HMGCR mRNA and protein and PCREB protein in ventricle tissue using real-time PCR and immunohistochemistry, respectively. Echocardiography was used to assess mouse heart function.

\section{Results}

(1) TSHR mRNA and protein expression was present in both the ventricle tissue and myocytes of rats and mice. TSHR protein was located in H9C2 cell membrane as same as FRTL-5 cells.

(2) bTSH stimulated the accumulation of cAMP production in H9C2 cells in dose-and time dependent manners (all $p<0.05$ ), indicating TSHR was functional in ventricle myocytes.

(3) Treatment with bTSH resulted an increase in either the expression of $\mathrm{pCREB}$ protein, HMGCR mRNA and protein or the BNP secretionin in dose- and time-dependent manners (all $p<0.05$ ).

(4) The above bTSH-stimulated effects were not only blunted by $\mathrm{SQ} 22536$ but also by $\mathrm{H} 89$, indicating TSH up-regulated HMGCR expression in ventricle myocytes through cAMP/PKA/ pCREB signalling pathway.

(5) The immunohistochemical results of mouse ventricle tissue showed that HMGCR protein mainly expressed in cytoplasm while pCREB protein expressed in cell nuclei. In comparison to wild type mice, the expression, in left ventricle of $\mathrm{Tshr}^{-/-}$type mice, of HMGCR mRNA and protein and pCREB protein was significantly reduced by $15-36 \%(p<0.05)$. The results from Echocardiography demonstrated there was no significant difference in LVESD, LVEDD, LVPWT, EF and FS between the two type mice ( $p>0.05)$.

Conclusions Functional TSHR exists in ventricle tissue and myocytes. TSH induces ventricle myocyte HMGCR expression through cAMP/PKA/pCREB and promotes BNP secretion, which is dependent of TSHR. 